\title{
XXIX. Zur Kenntnis der Beziehungen zwischen chemischer Konstitution und Krystallstruktur.
}

\author{
Von
}

P. Groth in München.

Im 1. Hefte dieses Bandes (S. $65 \mathrm{f.}$ 1)) wurde aus den Resultaten der Untersuchung der Krystalle durch die Interferenz der Röntgenstrablen folgender Schluß gezogen: die Krystalle sind aus den Atomen derart zusammengesetzt, daß die Orte, um welche die letzteren ihre Wărmebewegungen ausführen, ein aus einer Anzahl ineinander gestellter kongruenter Raumgitter bestehendes regelmäßiges Punktsystem bilden, welches dem Gleichgewichte der von den Atomen aufeinander ausgeübten Kräfte entspricht. Der prinzipielle Gegensatz, in welchen dadurch die Krystalle zu den aus Molekülen bestehenden Stoffen, den Gasen, Flüssigkeiten, Gelen und den sogenannten amorphen festen Körpern, treten, zeigt sich in ihrem thermischen Verhalten. Der Übergang , fest $\rightleftarrows$ flüssig ^ findet im Falle einer krystallisierten Substanz bei einer bestimmten Temperatur, dem Schmelz- bezw. Erstarrungspunkte, unter diskontinuierlicher Änderung der Eigenschaften und unter Bindung bezw. Freiwerden von Wärme statt, im Falle einer amorphen Substanz dagegen allmählich und ohne Wärmetönung. Ferner nimmt nach den Untersuchungen von Eucken ${ }^{2}$ ) die

1) S. 66 des zitierten Aufsatzes wurde von SchoenflieB' Theorie der Krystallstruktur behauptet, daß sie den Erfahrungen an den optisch aktiven Substanzen widerspreche. Durch persönliche Besprechung mit Herrn Schoenfließ habe ich mich bald darauf davon überzeugt, da $B$ jene Behauptung auf einem Mißverständnisse meinerseits beruhte. Das Resultat dieser Besprechung war der Entschluß des Herrn SchoenCließ, eine Darstellung des Verhältnisses seiner Theorie zu den Anschauungen abzufassen, welche sich neuerdings aus der experimentellen Erforschung der Krystallstruktur ergeben haben. Eine den ersten Teil dieser Darstellung umfassende Abhandlung ist während der Drucklegung des vorliegenden Heftes eingegangen und wird im nächsten Hefte erscheinen.

2) S. diese Zeitschr. 54, 410 f. 
Wärmeleitfähigkeit der Krystalle mit abnehmender Temperatur stark zu, während sie bei den amorphen festen Körpern (Gläsern) abnimmt; der Umstand, daß der absolute Wert der Leitfähigkeit sich für krystallinische Aggregate kleiner ergibt, als für Krystalle, beweist, daß Unregelmäßigkeit der Struktur die Fähigkeit, Wärme zu übertragen, verringert. Diese Ergebnisse stehen im vollkommenen Einklange mit den Resultaten der Erforschung der Krystallstruktur, denn die Atome eines Krystalles führen bei dem absoluten Nullpunkte der Temperatur keinerlei Wärmebewegungen mehr aus, stellen also dann den höchsten Grad von Regelmäßigkeit der betr. Substanz dar, während die Beschaffenheit der amorphen Körper auch in den niedrigsten Temperaturen eine unregelmäßige sein muß.

Was die Bindung der Atome in der Krystallstruktur betrifft, so ist für den Diamanten nachgewiesen worden (siehe l. c. 70), daß dieselbe erklärt werden könne durch die Valenzen des Kohlenstoffs; bei den übrigen bisher untersuchten krystallisierten Substanzen ist dies z. Z. noch nicht möglich, wenigstens haben Versuche des Verf.'s, mit Hilfe von Modellen die Krystallstruktur derselben durch Bindungen, welche den Valenzen der betr. Atome entsprechen, zur Darstellung zu bringen, bisher noch keine befriedigenden Resultate geliefert. Während außerdem beim Diamanten die Anordnung der Atome eine Beziehung zu der oktaëdrischen Spaltbarkeit deutlich erkennen läßt (s. l. c.), ist dies bei anderen Körpern nicht der Fall, und die Abhängigkeit der Anordnung von der Natur der Atome, deren gegenseitige Einwirkung sich durch die Kohäsion und andere vektorielle Eigenschaften äußert, bleibt noch zu erforschen. Jedenfalls müssen wir aber das "Atom *, $\mathrm{da}$ es nach verschiedenen Richtungen im allgemeinen verschiedene Kräfte ausübt, als ein anisotropes Gebilde betrachten; ebenso müssen die Moleküle der amorphen Körper, da sie aus den Atomen in bestimmter Weise aufgebaut sind, anisotrop sein; die Isotropie der amorphen Körper, d. h. ihr gleiches Verhalten in verschiedenen Richtungen, kann daher nur durch die Regellosigkeit der gegenseitigen Orientierung der Moleküle bedingt sein, derzufolge bei ihren selbst gegen die Wellenlängen des Lichtes kleinen Dimensionen die Messung des Zahlenwertes einer Eigenschaft stets zu einem mittleren (und daher immer glcichen) Wert, unabhängig von der Richtung, führt.

Die Anisotropie der Moleküle bedingt notwendig, daß sie bei genügender gegenseitiger Annäherung richtende Kräfte aufeinander ausüben, denen zufolge sie sich in bestimmter Weise gegeneinander orientieren, sei es in paralleler Stellung, sei es in einer anderen Orientierung ( $Z$ willingsstellung), in welcher Gleichgewicht der Molekularkräfte stattfindet. Diese Richtkräfte werden aber naturgemäß um so schwächer sein, je geringer der Grad der Anisotropie der Moleküle, d. b. je größer das Molekulargewicht ist, und damit dürfte der Umstand zusammenhängen, $\mathrm{da} ß$ die Kolloide und manche 
der in neuester Zeit, besonders von E. Fis cher ${ }^{1}$ ) dargestellten Verbindungen, deren Moleküle aus einer außerordentlich großen Zahl von Atomen bestehen, nur in amorphem Zustande erhalten werden können. Sind jene Kräfte ausreichend, die Parallelstellung der Moleküle zu bewirken und auch bei einer Aufnahme kleinerer Moleküle, z. B. denen des Wassers, in die Zwischenräume zù erbalten, so muß ein anisotropes Gebilde entstehen, welches der Quellung fähig ist und eine verschwindend kleine Kohäsion zeigt; vielleicht liegt hierin die Erklärung der optischen Eigenschaften der »flüssigen Krystalle «, der quellbaren $»$ Krystalloide* der Pflanzensamen und derjenigen Kieselgele, die sich bei der Zersetzung gewisser Silikate durch Säuren bilden (in letzterem Falle wäre natürlich die regelmäßige Orientierung der Gelmoleküle durch die Stellung der $\mathrm{Si}$ - und $\mathrm{O}$-Atome in der Krystallstruktur des ursprünglichen Minerals bewirkt).

Krystallisation, d. h. Bildung einer regelmäßigen Anordnung der Atome, kann, da hier lediglich Atomkräfte in Frage kommen, nur dadurch zustande kommen, daß wenigstens ein Teil der innerhalb jedes Moleküls bestehenden Bindungen in solche zwischen Atomen benachbarter Moleküle, deren gesonderte Existenz damit aufhört, übergeht - und offenbar muß es von der Struktur der betreffenden Moleküle abhängen, welche ihrer Atombindungen in dem neuen Gleichgewichtszustande erhalten bleiben und welche durch andere ersetzt werden. Daß in der Tat ein Teil der im Moleküle vorhandenen inneren Bindungen auch in den Krystallbau übergeht, beweisen die mannigfachen bisher erkannten Beziehungen zwischen der Struktur des chemischen Moleküls und der Krystallstruktur bezw. der Krystallform. So hat nach dem Pasteurschen Gesetze die Enantionorphie des chemischen Moleküls stets die gleiche Eigenschaft für den Krystallbau zur Folge. Ferner geht das Fortbestehen gewisser intramolekularer Atombindungen in der Krystallstruktur daraus hervor, daß die letztere häufig Symmetrieverhältnisse zeigt, welche unzweifelhaft schon im chemischen Molekül vorhanden waren. Auf solche Fälle ist früher schon mehrfach hingewiesen worden, und sie sind durch besonders wichtige vermehrt worden bei den Untersuchungen Wahl's ${ }^{2}$ ) über Krystallisation bei tiefen Temperaturen; durch letztere ist nachgewiesen worden, daß die Ḱrystallsymmetrie der einfachsten Kohlenstoffverbindungen im allgemeinen durch die Symmetriebedingungen ihres chemischen Moleküls bestimmt wird.

Im Zusammenhange damit steht auch die Tatsache, daß in zahlreichen Fällen der Ersatz eines Atoms durch ein anderes oder eine Atomgruppe eine Änderung der Krystallstruktur nur in einer bestimmten Richlung bewirkt, daß also eine Verwandtschaft der Struktur der chemischen Moleküle

1) Ber. d. d. chem. Ges. $1913,46,3287$.

2) Zeitschr. f. physik. Chemie 1914, 88, 154 f. 
Zur Kenntnis d. Beziehungen zwischen chem. Konstitution u. Krystallstruktur. 501

zweier Substanzen teilweise auch in der Krystallstruktur erhalten bleibt. Diese (morphotropischen) Beziehungen wurden zuerst an den aromatischen Körpern nachgewiesen und sind hier unverhältnismäßig viel häufiger, als bei den aliphatischen Verbindungen, ein Umstand, der vermuten läßt, daß die Ringbindung der Kohlenstoffatome in den Benzolderivaten besonders geeignet sei, aus dem Bau des Moleküls in denjenigen des Krystalls überzugehen. Daß sogar die Verschiedenheit der Äthylen- und Acetylenbindung von der einfachen Bindung zweier Kohlenstoffatome an Wichtigkeit für die Krystallstruktur gegenüber der cyclischen Bindung zurücktreten kann, lehrte der von Boeris ${ }^{1}$ ) geführte Nach weis einer sehr nahen krystallographischen Verwandtschaft der folgenden drei Substanzen:

$$
\begin{aligned}
& \text { Dibenzyl }=\mathrm{C}_{6} \mathrm{H}_{5} \cdot \mathrm{CH}_{2} \cdot \mathrm{CH}_{2} \cdot \mathrm{C}_{6} \mathrm{H}_{5} \\
& \text { Stilben }=\mathrm{C}_{6} \mathrm{H}_{5} \cdot \mathrm{CH}: \mathrm{CH} \cdot \mathrm{C}_{6} \mathrm{H}_{5} \\
& \text { Tolan }=\mathrm{C}_{6} \mathrm{H}_{5} . \mathrm{C}: \mathrm{C} . \mathrm{C}_{6} \mathrm{H}_{5} .
\end{aligned}
$$

Alles dies legte die Frage nahe, ob auch andere ringförmige Atombindungen, als sie im Benzolkern vorliegen, einen ähnlich weitgehenden Einfluß auf die Krystallstruktur ausüben, z. B. die Ringschließung durch cin Saucrstoffatom oder durch die Iminogruppe $N H$ bei zweibasischen aliphatischen Säuren.

In der Tat zeigen nun, während Bernsteinsäure und Maleïnsäure (beide monoklin) kcine näheren krystallographischen Beziehungen erkennen lassen, ihre beiden rhombisch krystallisierenden Anhydride

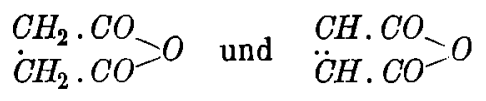

eine sehr nahe Verwandtschaft ihrer Formen, auf welche schon vor langer Zeit Bodewig ${ }^{2}$ ) aufmerksam machte; die neuerdings durch Steinmetz i. Lab. d. Verf.'s vorgenommene Bestimmung des spez. Gewichts der Maleïnsäure $=1,609$ und ihres Anhydrids $=1,509$ gestattet nun, die Beziehungen dieser Körper durch Berechnung der topischen Parameter näher festzustellen; diese Werte betragen für die Säuren:

Bernsteinsäure: $\chi: \psi: \omega=3,413: 5,940: 5,097 ; \beta=133^{\circ} 37^{\prime}$

Maleïnsãure: $\quad \chi: \psi: \omega=3,977: 5,384: 3,783 ; \beta=1177$.

Dagegen für die Anhydride:

Bernst.-Anhydrid: $\chi: \psi: \omega=3,711: 6,242: 2,873 ; \beta=90^{\circ}$

Maleïns.- $\gg \chi: \psi: \omega=3,814: 5,952: 2,854 ; \beta=90$.

Die Imide können nicht verglichen werden, da dasjenige der Maleïnsăure nicht existenzfähig ist, und da auch die Halogen- und Oxyderivate

1) Diese Zeitschr. 1901, 34, 298.

2) Diese Zeitschr. 1881, 5, 573. 
der beiden Săuren der Untersuchung nach dieser Richtung hin Schwierigkeiten in den Weg stellen, so veranlaßte der Verf. zur weiteren Prüfung vorliegender Frage die Untersuchung einer Anzahl homologer Verbindungen, von deren einfacheren nur eine unvollständige Bestimmung der Monomethylbernsteinsäure (Brenzweinsäure) vorlag. Es wurden durch Stefl1) untersucht: die beiden symmetrischen Dimethylbernsteinsäuren und die asymmetrische, die Trimethylbernsteinsăure, die Tetrametbylbernsteinsäure, die symmetrische Dimethyldiäthylbernsteinsäure, sowie eine Anzahl von ihren Anhydriden und Imiden, während Drugman ${ }^{2}$ ) die Untersuchung anderer zweibasischer Säuren, der Malonsäure, der Dimethyl- und Diäthylmalonsäure, der Glutarsäure, Dimethylglutarsäure u. a. ausführte.

Yon allen diesen Säuren lassen die einander am nächsten stehenden, d. h. nur durch ein oder zwei Methylgruppen unterschiedenen, keinerlei nähere morphotropische Beziehungen erkennen; nur zwei einander ferner stehende, die trans-symmetrische Dimethylbernsteinsäure und die $n$-symmetrische Dimethyldiäthylbernsteinsäure lassen sich auf ähnliche Axenverhältnisse zurückführen, zeigen aber einen recht verschiedenen Krystallhabitus.

Geht man jedoch von den Säuren zu den Anhydriden über, so lehrt die Vergleichung der einander nächst stehenden Verbindungen, daß der Eintritt der $\mathrm{CH}_{3}$-Gruppe eine nur in bestimmter Richtung stattindende Änderung in der Krystallstruktur bewirkt hat.

Nach Stefl (1. c. 359 f.) sind die Elemente von

Bernsteinsäureanhydrid (rhomb.): $\quad a: b: c=0,5945: 1: 0,4603 ; \beta=90^{\circ}$ $s$-Dimethylbernsteins.-Anhydrid(mon.): $a: b: c=0,3859: 1: 1,6044 ; \beta=93^{\circ} 31^{\prime}$

Die Substitution von $H$ durch $C H_{3}$ hat also außer der die Regel bildenden Erniedrigung der Symmetrie nur die $c$-Axe wesentlich geändert (wegen deren großen Verschiedenheit eignen sich in diesem Falle die topischen Parameter weniger gut zur Vergleichung) $\left.{ }^{3}\right)$.

Ähnliche Verhältnisse zeigen die Imide (1. c. 365 f.), nämlich:

Bernsteinsäureïmid (rhomb.): $\quad a: b: c=0,7888: 1: 1,3655 ; \beta=90^{\circ}$ $s$-Dimethylbernsteinsäureïmid(mon.): $a: b: c=0,9089: 1: 1,3153 ; \beta=100 \quad 10 \frac{1}{2}^{\prime}$.

1) Diese Zeitschr. 54, $343 \mathrm{f}$.

2) Diese Zeitschr. 53, $240 \mathrm{f}$.

3) S. 362 der zilierten Arbeit beschreilt St efl Krystalle von $h$-symmetr. Dimethyldiäthylbernsteinsäureanhydrid, und S.370 solche von dem entsprechenden Imid, welche aulfallenderweise die gleiche Form zeigten. Nach dem von $S$ te fl (S. 9 seiner chem. Dissert. d. techn. Hochsch. Muinchen 1914) angegebenen Verfahren entsteht aber, wie Ste in metz neuerdings fand, nicht das Anhydrid, sondern das Imid (beide haben nabezu den gleichen Kohlenstoffgehalt, mit dessen Bestimmung sich $S t e f l$ bei seinem vermeintlichen Anhydrid begnügte, obgleich er für dasselbe die gleiche Krystallform und den gleichen Schmelzpunkt fand, wie für das Imid). 
Zur Kenntnis d. Beziehungen zwischen chem. Konstitution u. Krystallstruktur. 503

Hier ist also das Verbăltnis $b: c$ nahe konstant geblieben, und die Verwandtschaft läßt sich auch aus den topischen Parametern ersehen:

Bernsteinsäureïmid: $\chi: \psi: \omega=3,193: 4,048: 5,527 ; \beta=90^{\circ}$

$s$-Dimethylverb.: $\quad \chi: \psi: \omega=3,982: 4,381: 5,762 ; \beta=100101^{\prime}$.

Größere Veränderungen bringt der Eintritt weiterer Methylgruppen hervor, aber die pseudorhombische Bipyramide $\{111\},\{111\}$ des Tetramethylbernsteinsäureïmids hat noch eine deutliche Verwandtschaft mit der Bipyramide des Bernsteinsăureïmids bewahrt, wie aus den topischen Parametern hervorgeht, sobald man die Werte $\chi$ und $\psi$ der Tetramethylverbindung vertauscht, alsdann wird

$$
\chi: \psi: \omega=3,415: 5,425: 7,063 ; \quad \beta=92^{\circ} 8^{\prime} .
$$

Man sieht hieraus, daß die Strukturdimensionen durch die Aufnahme von vier Methylgruppen in den Krystallbau nach der $a$-Axe nur wenig, nach $\operatorname{der} b$ - und $c$-Axe mehr, aber ungefähr in gleichem Verhältnisse zugenommen haben. Durch den nochmaligen Eintritt von vier Methylgruppen schreitet die Abnahme der Symmetrie noch weiter fort, denn Tetraäthylbernsteinsäureïmid krystallisiert triklin, aber seine topischen Parameter:

$$
\chi: \psi: \omega=4,556: 5,329: 7,937
$$

lassen immer noch eine gewisse Verwandtschaft mit den vorhergehenden Gliedern dieser homologen Reihe erkennen.

Was für die Homologen der Bernsteinsäure gilt, schcint auch bei denen der Maleïnsäure Geltung zu behalten, denn es wurde schon früher darauf hingewiesen ${ }^{1}$ ), daß das Anhydrid der Dimethylmaleïnsäure mit demjenigen der Maleïnsäure selbst Gleichheit der Symmetrie und Ähnlichkeit der Winkel in je einer der herrschenden prismatischen Zonen besitze. Eine noch größere Ähnlichkeit zeigt ersteres mit dem Anhydrid der $s$-Dimethylbernsteinsäure, sodaß hier ein Fall vorliegt, wie bei Dibenzyl und Stilben (S. 501):

$$
\begin{aligned}
& \mathrm{CH}_{3} \cdot \mathrm{CH} \cdot \mathrm{CO}>\mathrm{O} \text { und } \begin{array}{l}
\mathrm{CH}_{3} \cdot \mathrm{C} \cdot \mathrm{CO} \\
\mathrm{CH}_{3} \cdot \mathrm{CH} \cdot \mathrm{CH} \cdot \mathrm{CO}
\end{array}=\mathrm{O} . \mathrm{OO}
\end{aligned}
$$

Wie S. 502 erwähnt, liegen von einer Reihe anderer zweibasischer Säuren nunmehr neue Bestimmungen vor, nach denen ebenfalls die einander zunächst stehenden homologen keine nähere krystallochemische Verwandtschaft zeigen. Solche ist nach dem vorhergehenden zu erwarten bei den Anhydriden und Imiden, von denen aber nur diejenigen der Glutarsäure

1) Siehe P. Groth, Chem. Kryst. 3, 465. Ebenda S. 283 wurde auch auf die merkwürdige Ähnlichkeit der Krystallformen von Dibrommaleïnsäureïmid und Dibrom. succinimid, sowie S. 406 auf die krystallochemische Verwandtschaft von Citraconsäureanhydrid mit Maleïnsäure- und Itaconsäureanhydrid aufmerksam gemacht. 
untersucht sind, zwischen denen Ähnlichkeit der Winkel je einer prismatischen Zone besteht, während sie in den Kohäsionsverbältnissen völlig voneinander abweichen. Die Untersuchungen bomologer Verbindungen würden daher wohl keine näheren Beziehungen zwischen den Anhydriden einerseits und den Imiden andererseits, wohl aber solche zwischen den Gliedern je einer dieser beiden Reihen zu Tage fördern.

Bei den zweibasischen Säuren von der bisher besprochenen Art kommt eine ringförmige Bindung auch dadurch zustande, daß die Wasserstoffatome der beiden Carboxylgruppen durch ein zweiwertiges Metall vertreten werden. Um Beziehungen zwischen derartigen Salzen verwandter Sãuren festzustellen, müßten wasserfreie Salze oder solche mit gleichem Wassergehalt verglichen werden, wozu das erforderliche Beobachtungsmaterial noch nicht vorliegt.

Da das Glykokoll sich tautomer als eine cyclische Verbindung verhält, so wäre es von Interesse, die krystallochemischen Beziehungen der Methylderivate der Aminoessigsäure bis zu dem unzweifelhaft cyclischen Betaīn zu untersuchen.

Aus dem Vorstehenden ist ersichtlich, daß auch unter den aliphatischen Körpern sich Reihen finden, welche ganz ähnliche Verbältnisse darbieten, wie die aromatischen, $d . h$. daß auch bei diesen eine vorbandene Ringbindung der Atome aus der Struktur des Moleküls in den Krystallbau übergehen kann, daß daher auch deren Untersuchung zur Erforschung des Zusammenhanges zwischen der chemischen Konstitution und der Krystallstruktur beitragen kann. In weit höherem Grade bieten außer den Benzolderivaten dafür das Material dar die aromatisch-heterocyclischen Verbindungen, in denen Kohlenstoff- und Stickstoflatome ringförmig verbunden sind und über welche zwar viele krystallographische Einzelbestimmungen, aber noch keine systematischen Untersuchungen zusammenbängender Reihen von Substanzen existieren. 